\title{
Cyclohexene Photo-oxidation
}

over Vanadia Catalyst Analyzed by Time Resolved ATR-FT-IR Spectroscopy

\author{
Guido Mul*a ${ }^{\star a}$ Walter Wasylenko ${ }^{b}$, M.Sameh Hamdy ${ }^{a}$, and Heinz Frei ${ }^{\star^{b}}$ \\ ${ }^{a}$ Catalysis Engineering (CE), DelftChemTech, \\ Technische Universiteit Delft, Julianalaan 136, 2628 BL, Delft, The Netherlands. \\ ${ }^{b}$ Physical Biosciences Division, Lawrence Berkeley National Laboratory, University of \\ California, Berkeley, California, CA 94720
}

${ }^{*}$ Corresponding Authors

\begin{abstract}
Vanadia was incorporated in the 3-dimensional mesoporous material TUD-1 with a loading of $2 \% \mathrm{w} / \mathrm{w}$ vanadia. The performance in the selective photo-oxidation of liquid cyclohexene was investigated using ATR-FT-IR spectroscopy. Under continuous illumination at $458 \mathrm{~nm}$ a significant amount of product, i.e. cyclohexenone, was identified. This demonstrates for the first time that hydroxylated vanadia centers in mesoporous materials can be activated by visible light to induce oxidation reactions. Using the rapid scan method, a strong perturbation of the vanadyl environment could be observed in the selective oxidation process induced by a $458 \mathrm{~nm}$ laser pulse of $480 \mathrm{~ms}$ duration. This is proposed to be caused by interaction of the catalytic centre with a cyclohexenyl hydroperoxide intermediate. The restoration of the vanadyl environment could be kinetically correlated to the rate of formation of cyclohexenone, and is explained by molecular rearrangement and dissociation of the peroxide to ketone and water. The ketone diffuses away from the active center and ATR infrared probing zone, resulting in a decreasing ketone signal on the tens of seconds time scale after initiation of the photoreaction. This study demonstrates the high potential of time resolved ATR FT-IR spectroscopy for mechanistic studies of liquid phase reactions by monitoring not only intermediates and products, but by correlating the temporal behavior of these species to molecular changes of the vanadyl catalytic site.
\end{abstract}

Keywords: Time resolved spectroscopy, cyclohexene, oxidation, cyclohexenyl hydroperoxide, ATR spectroscopy, infrared, vanadia, silica, TUD-1, photocatalysis 


\section{Graphical Abstract}

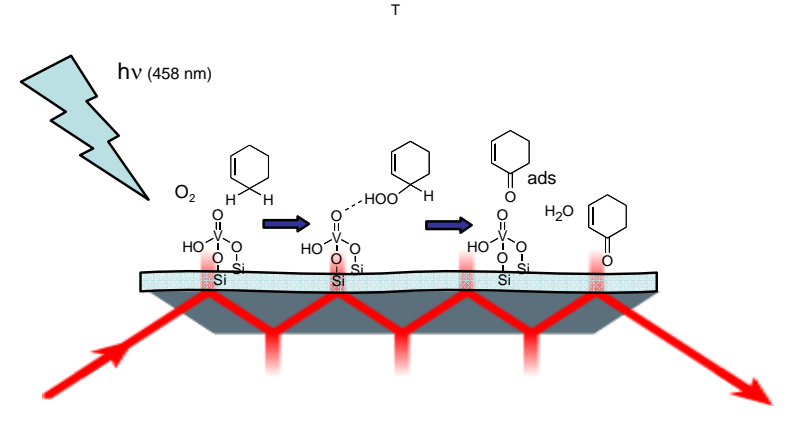

ATR analysis of vanadia catalyzed photon induced oxidation of cyclohexene to cyclohexenone 


\section{Introduction}

Supported vanadium oxides are effective photocatalysts in selective hydrocarbon oxidation, both in gas phase applications [1-3] as well as in liquid phase [4].Typically the active vanadia sites are completely dehydrated during reaction and, without promoters, UV radiation is required to photo-activate the catalytic centers $[2,3]$. Generally water is reported to deteriorate the photocatalytic performance of vanadia catalysts [5] even though hydration shifts the absorption spectrum of these catalysts into the visible $[6,7]$. Various groups have postulated a mechanism for the photocatalytic action of vanadia, but little in situ spectroscopic evidence is available to describe the pathway of the molecular transformations of hydrocarbons in contact with the vanadia sites, or potential structural rearrangements of the vanadia site upon light activation, reaction, and (de)hydration.

In the present contribution we report an in situ time resolved ATR-FT-IR spectroscopy study of cyclohexene oxidation over vanadia centers incorporated in the novel mesoporous material TUD-1. The infrared data show that visible light induced selective photo-oxidation reactions over vanadia centers are possible without promoters, if the vanadia centers are hydroxylated. Experiments directly reveal the involvement of a cyclohexenyl hydroperoxide intermediate in the selective formation of cyclohexenone. Our study furthermore shows the high potential of time resolved ATR FT-IR spectroscopy in analyzing liquid phase photooxidation reactions by monitoring not only the dynamics of reactants and products, but that of the catalytic metal site as well.

\section{Experimental}

\subsection{Material synthesis}

V-TUD-1 was synthesized with a loading of $2 \% \mathrm{w} / \mathrm{w}$ in a one-pot surfactant-free procedure using triethanolamine (TEA) as a bi-functional template and tetraethyl ammonium hydroxide (TEAOH) as a base [8]. A mixture of $15.1 \mathrm{~g}$ of TEA (97\%, ACROS) and $6.5 \mathrm{~g}$ of deionized water was added drop-wise into a mixture of $20.9 \mathrm{~g}$ tetraethylorthosilicate (TEOS, $98 \% \mathrm{ACROS}$ ) and the vanadia salt solution ( $0.5 \mathrm{~g}$ of Vanadium (V) triisopropoxide ( $\mathrm{VO}\left[\mathrm{CHO}\left(\mathrm{CH}_{3}\right)_{2}\right]_{3}$, Alfa Aesar) in $5 \mathrm{~mL}$ deionized water) while stirring. Once the addition was complete, the solution was stirred for an additional 5 minutes followed by drop wise addition of $12.4 \mathrm{~g}$ of TEAOH (35\%, Aldrich). Then the mixture was aged at room temperature for 24 $\mathrm{h}$, evaporated until dryness and treated at $373 \mathrm{~K}$ for $24 \mathrm{~h}$ in air, followed by hydrothermal treatment at $453 \mathrm{~K}$ for $8 \mathrm{~h}$ in a teflon-lined autoclave. Finally the obtained solid products were calcined in static air at $873 \mathrm{~K}$ for $10 \mathrm{~h}$ applying a ramp rate of $1 \mathrm{~K} / \mathrm{min}$. TUD-1 without 
vanadium was synthesized as described elsewhere [9], and was used to confirm that the vanadia centers are required to initiate the photon induced reaction.

\subsection{ATR Spectroscopy}

The ATR spectra were recorded with an ASI systems model Dicomp ATR accessory (PN 071-1213) equipped with a three reflection diamond ATR crystal and incorporated in the sample compartment of a Bruker Model IFS 66v/S spectrometer. The catalyst was deposited on the ATR crystal by suspension of $25 \mathrm{mg}$ of the ground catalyst (mortar) in $1 \mathrm{ml}$ of deionized water, followed by 10 minutes of sonication (VWR, model 50HT). Subsequently, a drop of 3 microliter of the suspension was deposited on the crystal, followed by evaporation to dryness by applying a reduced pressure of about 1 Torr. This procedure was repeated three times, after which an increase in intensity of the infrared bands due to the silica framework vibrations could no longer be observed (which signaled that sufficient silica material was deposited for optimal probing by ATR infrared). To prevent substantial dissolution of vanadia in water $[5,10]$, the coatings were applied immediately after preparation of the suspension.

A home built teflon liquid cell was assembled over the coated diamond crystal, followed by evacuation to a pressure of about 100 mTorr, sufficient to remove the majority of adsorbed water, as confirmed by the ATR spectra. Then a pressure of 50 Torr of cyclohexene was introduced to the catalyst layer and allowed to equilibrate for about 10 minutes. Finally oxygen (99\%, Matheson, used as received) was released in the cell compartment to atmospheric pressure, after which an aliquot of $0.2 \mathrm{ml}$ pure cyclohexene (Alpha Aesar, >99.5\%) was immediately introduced into the cell and closed off with a quartz plate to prevent re-adsorption of moisture on the catalyst surface and evaporation of cyclohexene. Prior to initiation of photo-catalyzed reaction, spectra were recorded over a period of 15 min to verify that no reaction occurred in the absence of photolysis light.

\section{Continuous illumination}

Chemical reaction was initiated by exposure of the catalyst-coated ATR crystal, without liquid agitation, to an Ar ion laser beam at $458 \mathrm{~nm}(160 \mathrm{~mW})$ (Coherent model Innova 90-6). The laser beam size was adjusted by lens optics so that it matched the size of the 5 $\mathrm{mm}^{2}$ diamond in the ATR top plate. Spectra were recorded after 30 second photolysis intervals. 


\section{Rapid scan experiments}

Rapid scan FT-IR measurements were conducted using the same ATR accessory (ASI systems) and Bruker spectrometer (Model IFS 66v/s) as outlined in the previous paragraph. A highly sensitive HgCdTe photon detector Kolmar model KMPV8-1-J2 with an 8 micron band gap was used. Reaction was initiated by visible light from an Ar ion laser (Coherent model Innova 90-6) equipped with a mechanical shutter (UniBlitz model D122). The emission wavelength was $458 \mathrm{~nm}$ and the pulse duration was $480 \mathrm{~ms}$ (160 $\mathrm{mW}$ ). The shutter was triggered by the forward motion of the interferometer mirror for precise timing of reaction initiation and start of data acquisition. After triggering of the shutter, the rapid scan spectrometer was collecting a large series of interferograms, split by macro software into lumps, containing a variable number of scans, representative for time slices at 122 ms, 366 ms, 793 ms, 1403 ms, 2013 ms, 2928 ms, 4453 ms, 6588 ms, $9333 \mathrm{~ms}$, and $11468 \mathrm{~ms}$ after the laser pulse (midpoints). A total of 10 such series, yielding 10 identical single beam spectra at each respective midpoint after Fourier transformation, was obtained by giving 10 laser pulses to the catalyst in one complete experiment. Final time-resolved spectra for a given time delay were obtained by computing the ratio of each of the 10 corresponding stored single beam spectra against the single beam spectrum taken just before the pulse. The 10 ratioed spectra were then averaged to yield the absorbance time slice for a given time delay. More details of the experimental procedure can be found in $[11,12]$.

\section{Results}

\subsection{Layer deposition and introduction of cyclohexene}

Spectra representative of the experimental steps before the start of the steady state and time resolved experiments are shown in Figure 1 . The spectrum of the $2 \%$ vanadia catalyst obtained after deposition of three layers is depicted in Figure 1a. Physisorbed water is apparent from the broad feature centered around $-3400 \mathrm{~cm}^{-1}$ (stretching mode) and the absorption at $1630 \mathrm{~cm}^{-1}$ (bending mode). The spectrum is dominated by the vibrations of the silica framework of the TUD-1 material in the range between $1300 \mathrm{~cm}^{-1}$ and $700 \mathrm{~cm}^{-1}$, with maxima located at 1078 (shoulder at $1200 \mathrm{~cm}^{-1}$ ), 963 and $797 \mathrm{~cm}^{-1}$. These bands have respectively been assigned to Si-O-Si (asymmetric stretch), Si-O-H (silanol nests), and Si-OSi (symmetric stretch) vibrations [13]. The spectral features are very similar to those reported for a thin film of a titania-silica aerogel catalyst on a ZnSe Internal Reflection Element (IRE) by Baiker and coworkers [13]. A large extent of hydration of the catalyst under ambient conditions is also apparent from the UV-Vis spectrum of the catalyst, shown as an insert in Figure 1. The assignments of the absorption bands in the UV-Vis spectrum are discussed in 
detail elsewhere [6,7]. Changes in the Infrared spectrum upon dehydration, induced by evacuation to 100 mTorr, are shown in the difference spectrum Figure $1 \mathrm{~b}$. The negative signals at $-3400 \mathrm{~cm}^{-1}$ and $1630 \mathrm{~cm}^{-1}$ indicate the removal of water. At the same time significant changes can be observed in the silica framework region at 1033 and $970 \mathrm{~cm}^{-1}$. Corresponding peak positions for depletion upon evacuation of neat TUD-1 are at 1023 and $963 \mathrm{~cm}^{-1}$, respectively. The frequency differences between $\mathrm{V}$-containing and plain TUD-1 point to contributions of $\mathrm{V}=\mathrm{O}$ and $\mathrm{Si}-\mathrm{OH}$ stretch modes associated with $\mathrm{V}$ sites. The $970 \mathrm{~cm}^{-1}$ depletion is most probably due to the condensation $\mathrm{Si}-\mathrm{OH}$ groups with $\mathrm{V}-\mathrm{OH}$ groups upon dehydration [7]. However, we have found that the exact positions of the minima of these two bands depend on the extent of dehydration upon evacuation. The variability is most likely due to contributions from other types of condensing silanol sites (e.g. silanol nests) that also absorb in the region around $1000 \mathrm{~cm}^{-1}$ [14].

The addition of 50 Torr of cyclohexene vapor induces the spectral changes observed in Figure 1c. The signals due to cyclohexene are located at $640,718,875,916,1015,1135$, 1438 and $\sim 1680 \mathrm{~cm}^{-1}$. The spectrum is similar to that reported by Baiker and coworkers [13]. Besides the bands of cyclohexene, an enhancement of the intensity of spectral features of the catalyst support is observed, which originates from the difference in the refractive index of vacuum compared to condensed cyclohexene (larger penetration depth of the evanescent wave in the coated layer [15]). Furthermore, a band at $\sim 1015 \mathrm{~cm}^{-1}$ which agrees with the position of a weak cyclohexene absorption is far too intense for attribution to the hydrocarbon alone [16]. Because $\mathrm{V}=\mathrm{O}$ modes of single $\mathrm{V}$ centers on dehydrated silica supports are known to absorb in the range $1030-1040 \mathrm{~cm}^{-1}$ [17], we propose that the intense $1015 \mathrm{~cm}^{-1}$ band formed upon adsorption of cyclohexene originates from enhancement of $\mathrm{V}=\mathrm{O}$ by interaction with the hydrocarbon molecule.

Upon exposure of $1 \mathrm{~atm}$ of oxygen gas, no significant spectral changes were noted. Similarly, subsequent addition of liquid cyclohexene yielded no new bands, indicating that cyclohexene and oxygen do not react spontaneously over V-TUD-1 catalyst at room temperature.

\subsection{Steady state illumination}

Difference IR spectra obtained upon steady state illumination are after 30, 60, 90 and 120 seconds are shown in Figure 2. For comparison, a reference spectrum of cyclohexenone is shown in the bottom trace from which a cyclohexene spectrum was subtracted, to simulate the spectral changes upon conversion of cyclohexene to cyclohexenone. Five phenomena dominate the spectra during illumination. First, a sharp band is observed to grow in at 1692 $\mathrm{cm}^{-1}$, accompanied by bands at 1385, 1210,1118, 875, and $758 \mathrm{~cm}^{-1}$, which can be assigned to the formation of cyclohexenone. Second, a band emerges at $1660 \mathrm{~cm}^{-1}$ that is not present 
in the spectrum of loaded cyclohexenone (bottom trace of Figure 2). We attribute the absorption to photochemically produced cyclohexenone interacting with vanadyl sites (see discussion below). Third, a very strong band grows in at $970 \mathrm{~cm}^{-1}$ accompanied by one centered at $\sim 1035 \mathrm{~cm}^{-1}$. In view of the spectra shown in Figure 1, these absorptions indicate rehydration of the catalyst. The odd shape of the band at $\sim 1035 \mathrm{~cm}^{-1}$ is probably the result of a negative contribution of the $1015 \mathrm{~cm}^{-1}$ band (Figure 1), which will be discussed later. Fourth, negative bands at the positions of cyclohexene are observed, which confirm that a significant amount of cyclohexene is consumed. Fifth, broad features in the region of 1550$1750 \mathrm{~cm}^{-1}$ indicate that the photooxidation produces water in agreement with the growing bands around $1000 \mathrm{~cm}^{-1}$ attributed to rehydration. The broadness of the band in the 1550$1750 \mathrm{~cm}^{-1}$ region cannot be assigned with certainty, but a possibility is a change in the shape and intensity of the silica overtone band in the $1600-1700 \mathrm{~cm}^{-1}$ region (compare Figure 1 ). We conclude that excitation of the V-TUD-1 catalyst at $458 \mathrm{~nm}$ induces photooxidation of cyclohexene, yielding cyclohexenone.

UV-Vis absorption spectra of completely dehydrated, isolated $(\mathrm{SiO})_{3} \mathrm{~V}^{\mathrm{V}}=\mathrm{O}$ (vanadate) centers supported on silica do not show any absorption at this wavelength longer than 400 $\mathrm{nm}$ [6]. On the other hand, the ligand-to-metal charge-transfer absorption of silica-supported $\checkmark$ centers with hydroxyl ligands is shifted to the red, with an absorption tail extending to 500 nm [7], see Figure 1. As is shown by the time-resolved experiment below, irradiation with visible light directly shows the reaction of hydroxylated $V$ sites, thus demonstrating that this chromophore is responsible for the observed cyclohexene photooxidation.

\subsection{Rapid scan experiments}

The results of the rapid scan experiments are represented by the spectra shown in Figures 3 and 4 . Figure 3 shows the first five time slices of a time-resolved run. The photolysis pulse of 480 ms duration encompasses the 122 and 366 ms spectra, hence depletion as well as growth of bands of these two time slices represents the cumulative effect of continued photolysis over the first few hundred ms. By contrast, the 793 ms spectrum and all subsequent traces reflect the decay or growth kinetics of intermediates and final products. The main spectral features at these early times are the sharp, decreasing cyclohexene bands, depletion of an $\mathrm{OH}$ band at $3630 \mathrm{~cm}^{-1}$, growth of a very broad $\mathrm{OH}$ absorption spanning from $3600-3300 \mathrm{~cm}^{-1}$, a $20 \mathrm{~cm}^{-1}$ red shift of the $\mathrm{V}=\mathrm{O}$ band (derivative shape with minimum at 1015 and maximum at $995 \mathrm{~cm}^{-1}$ ), and decrease of a broad band at $780 \mathrm{~cm}^{-1}$. None of these spectral changes are observed when conducting an identical photolysis experiment using plain TUD-1 instead of V-TUD-1, confirming that they are due to photoreaction of cyclohexene with $\mathrm{O}_{2}$ over $\mathrm{V}$ sites (however, cyclohexene depletion caused by a slight (2-3 degree) laser heating effect was noted for experiments with TUD-1). An 
important clue for identifying the intermediate formed in the first two time slices is the instant rise of the broad $\mathrm{OH}$ stretch $3600-3300 \mathrm{~cm}^{-1}$. The growth is not accompanied by an equally fast growth at $1630 \mathrm{~cm}^{-1}$ where the water bending mode would absorb. A much slower rise is observed in the $1650-1620 \mathrm{~cm}^{-1}$ region in later spectra, but at times when the $3600-3300$ $\mathrm{cm}^{-1}$ absorption already has reached its maximum intensity (366 ms). This implies that the $\mathrm{OH}$ stretch mode observed on the hundreds of ms time scale is not due to $\mathrm{H}_{2} \mathrm{O}$ product. $A$ possible alternative explanation could be a $\mathrm{H}$ bonded $\mathrm{V}-\mathrm{OH}$ group, but this is not plausible: $\mathrm{a}$ $\mathrm{H}$ bond of $\mathrm{V}-\mathrm{OH}$ to a hydrocarbon would be likely for the final product cyclohexenone, which is inconsistent with our observation that the $3400 \mathrm{~cm}^{-1}$ band disappears as cyclohexenone is formed. The most likely conceivable intermediate featuring an $\mathrm{OH}$ bond is a hydroperoxide. We propose that the broad $\mathrm{OH}$ stretch absorption originates from cyclohexenyl hydroperoxide because the intermediate converts to cyclohexenone and $\mathrm{H}_{2} \mathrm{O}$ (see below), the established rearrangement products of the hydroperoxide [18]. At the same time, the transient $\mathrm{V}=\mathrm{O}$ band at $995 \mathrm{~cm}^{-1}$ also reaches maximum intensity at $366 \mathrm{~ms}$ and before starting to decrease. These observations suggests that the $\mathrm{OH}$ group of cyclohexenyl hydroperoxide is $\mathrm{H}$-bonded to the vanadyl group, resulting in the red shift of the $\mathrm{V}=\mathrm{O}$ mode to $995 \mathrm{~cm}^{-1}$. Concurrent bleach at $3630 \mathrm{~cm}^{-1}$ where free $\mathrm{V}-\mathrm{O}-\mathrm{H}$ groups are known to absorb $[7,19]$ is attributed to the formation of an $\mathrm{H}$ bonding interaction with the hydroperoxide intermediate, thereby shifting the $\mathrm{V}-\mathrm{O}-\mathrm{H}$ mode below $3600 \mathrm{~cm}^{-1}$. The decrease at $780 \mathrm{~cm}^{-1}, \mathrm{a}$ region typical for $\mathrm{Si}-\mathrm{O}$ stretch modes, is most likely due to $\mathrm{Si}-\mathrm{O}$ stretch modes whose environment is perturbed by the structural change at the $V$ catalytic site.

Spectra of Figure 3 recorded after termination of the photolysis pulse $(793 \mathrm{~ms}, 1.4 \mathrm{~s}$ and $2.0 \mathrm{~s}$ ) exhibit growth at 1695, 1385, 1210, 1118, $875 \mathrm{~cm}^{-1}$ due to cyclohexenone. A peak at $1660 \mathrm{~cm}^{-1}$ is assigned to the $\mathrm{C}=\mathrm{O}$ mode of cyclohexenone interacting with the $\mathrm{V}$ site, and water co-product is observed by the growth of a shoulder between 1650 and $1610 \mathrm{~cm}^{-1}\left(\mathrm{H}_{2} \mathrm{O}\right.$ bending mode). The rate of cyclohexenone and $\mathrm{H}_{2} \mathrm{O}$ growth over the initial $2 \mathrm{~s}$ period after the photolysis pulse coincides with the rate of decrease of the $995 \mathrm{~cm}^{-1}$ band of the vanadyl cyclohexenyl hydroperoxide intermediate. We conclude that rapid scan spectroscopy over the initial $2 \mathrm{~s}$ period reveals the spontaneous dissociation of the cyclohexenyl hydroperoxide intermediate, interacting with the $\mathrm{V}=\mathrm{O}$ site, to cyclohexenone and $\mathrm{H}_{2} \mathrm{O}$.

A detailed look at spectra on the $2-10 \mathrm{~s}$ time scale (Figure 4) reveals two processes. One is manifested by the continued decrease of the $995 \mathrm{~cm}^{-1} \mathrm{~V}=\mathrm{O}$ site and recovery of the 1015 and $780 \mathrm{~cm}^{-1}$ absorptions, which shows that vanadyl catalytic sites are restored as the cyclohexenyl hydroperoxide intermediate converts to cyclohexenone and water. At the same time, the cyclohexenone bands and the $\mathrm{H}_{2} \mathrm{O}$ bend absorptions start to decrease, indicating that product molecules inside the liquid cyclohexene-filled mesoporous support diffuse away from areas probed by the ATR infrared beam. In addition, a new absorption at $970 \mathrm{~cm}^{-1}$, only 
discernible as a shoulder in the first few seconds emerges as a distinct band in the last time slice at $9.3 \mathrm{~s}$. The absorption coincides with a decreasing band noted upon dehydration of $\mathrm{V}$ TUD-1 by evacuation (Figure 1, trace (b)) and is attributed to the Si-O stretch of $\mathrm{Si}-\mathrm{OH}$ groups formed upon hydrolysis of $\mathrm{V}-\mathrm{O}-\mathrm{Si}$ bonds by reaction with $\mathrm{H}_{2} \mathrm{O}$ co-product [7]. In fact, the hydrolyzed vanadyl site continues to grow under prolonged (steady state) photolysis (Figure 2). It is conceivable that, in addition, product water molecules enter the coordination sphere of the $V$ site. Because of the accumulation of adsorbed water on the catalyst surface, the time-resolved photolysis experiment shown in Figures 3 and 4 could be repeated about five times with the same sample, after which cyclohexenone formation was significantly suppressed. This is in agreement with earlier studies on vanadium based photocatalysis $[1,2]$ which indicate that water is diminishing catalyst performance. As a final observation, the cyclohexene bands recover (Figure 4) because the reactant depleted by photoreaction inside the silica mesopores is replenished by diffusion after the photolysis pulse is over.

\section{Discussion}

\subsection{Catalytic cycle}

In the present study we have shown that visible light induced photocatalytic oxidation of cyclohexene is possible over TUD-1 supported vanadia catalyst, which in view of the spectroscopic evidence contains at least one hydroxyl group. Not only have we been able to monitor the reaction intermediates and products, but also the direct involvement of the vanadium site. The proposed mechanism of the reaction is shown in Figure 5. The starting point for the reaction is the hydroxylated vanadyl site with adsorbed cyclohexene and oxygen. The steps involving the formation of the hydroperoxide occur at reaction rates which are significantly faster than the ms time resolution of the rapid scan experiments discussed here. While these steps therefore cannot be resolved, it is clear that peroxide formation does involve a catalytic function of the vanadia center, since in the absence of vanadia, products of oxidation were not observed. The Ligand to Metal Charge Transfer (LMCT) transition induces the formation of $\mathrm{OOH}$ and cyclohexenyl radicals, which recombine to form the adsorbed cyclohexenyl hydroperoxide intermediate. Rather than undergoing intermolecular reactions, we propose an intramolecular cyclohexenyl hydroperoxide rearrangement in which water is eliminated under formation of cyclohexenone within a few seconds. On a slower, tens of $s$ time scale, cyclohexenone product diffuses into solution (and away from the ATR infrared probing zone) while water molecules hydrate the $\mathrm{V}$ centers, ultimately causing deactivation.

Vanadium compounds have been widely used for the oxidation of olefins $[18,20,21]$, in particular with hydroperoxides as oxidants. Vanadia systems typically act as epoxidation 
catalysts, yet the epoxide was not observed in the present photocatalytic study. This is most likely the result of intramolecular rearrangement of the hydroperoxide to the ketone facilitated by interaction with the $\mathrm{V}$ center, rather than intermolecular reaction of the peroxide intermediate with another cyclohexene molecule.

\subsection{Nature of the active site.}

Based on recent literature, there is still no consensus on the precise structure of vanadia centers on silica matrices. While isolated centers are the most likely, recently it has been challenged whether $\mathrm{VO}_{4}$ units are attached with three legs to the matrix, or upsidedown with only one $[7,22]$. Furthermore the interpretation of vibrational spectra of this system is still under discussion, and new insights have been published recently $[7,22,23]$. There is consensus, however, that hydration of vanadia on silica can occur and is evidenced by a distinct spectroscopic signature. As indicated in the proposed mechanism shown in Figure 5, the postulated reactive vanadia center is partially hydroxylated. Evidence is based on the observation of transient loss of $\mathrm{V}-\mathrm{O}-\mathrm{H}$ absorption upon photoreaction and the fact that hydroxylated vanadyl centers absorb in the blue spectral region while isolated vanadyl groups in a completely dehydrated state do not. On the other hand, it has been reported that vanadia centers in the hydrated state are not active in photo-oxidation reactions $[3,24]$. This is confirmed in the present study, since accumulation of water on the catalyst leads to a significantly lower activity in the photo-oxidation of cyclohexene. Apparently, partial hydroxylation does not lead to inactive catalysts, and a single hydroxyl group appears most likely to be present in our catalytic system while more extensive hydroxylation deactivates the $V$ site. Future effort will seek reaction conditions, e.g. operation at slightly elevated temperature, that maintain a steady state hydroxylation level suitable for sustained reactivity.

\section{Conclusions}

In this paper we have demonstrated for the first time that activation of hydroxylated vanadyl centers on a mesoporous silica support with visible light leads to selective photooxidation of cyclohexene to cyclohexenone. The involvement of the vanadyl centers was established by spectral changes observed in time resolved rapid scan ATR experiments. The extent of hydration is a crucial factor in determining the activity of silica supported vanadyl centers: hydroxylation shifts the absorption spectrum to the visible, while progressive water accumulation on the catalyst surface deteriorates activity. Time resolved ATR studies prove to be an excellent means to analyze the complex reaction mechanism of liquid phase photocatalytic reactions. Improvement of the sensitivity of the method will open up recording 
at higher time resolution, which is required for monitoring elementary steps leading to formation of the hydroperoxide reaction intermediate.

\section{Acknowledgements}

This work was supported by the Director, Office of Science, Office of Basic Energy Sciences, Division of Chemical, Geological and Biosciences of the U.S. Department of Energy under Contract No. DE-AC02-05CH11231. Prof. Roger Sheldon, Delft University of Technology, is gratefully acknowledged for fruitful discussions regarding the mechanism of cyclohexenyl-hydroperoxide decomposition. STW is gratefully acknowledged for financial support of GM in the framework of the VIDI program. The authors thank Dirk Renckens for his contribution to the graphical abstract.

\section{References}

[1] Amano, F., Tanaka, T., and Funabiki, T. Langmuir 2004, 20, 4236-4240.

[2] Amano, F., Yamaguchi, T., and Tanaka, T. J. Phys. Chem. B 2006, 110, 281-288.

[3] Takenaka, S., Tanaka, T., Funabiki, T., and Yoshida, S. J. Chem. Soc. Faraday Trans. 1997, 93, 4151-4158.

[4] Teramura, K., Tanaka, T., Kani, M., Hosokawa, T., and Funabiki, T. J. Mol. Catal. A: Chemistry 2004, 208, 299-305.

[5] Shiraishi, Y., Morishita, M., Teshima, Y., and Hirai, T. J. Phys. Chem. B 2006, 110, 6587-6594.

[6] Gao, X. T., Bare, S. R., Weckhuysen, B. M., and Wachs, I. E. J. Phys. Chem. B 1998, 102, 10842-10852.

[7] Keller, D. E., Visser, T., Soulimani, F., Koningsberger, D. C., and Weckhuysen, B. M. Vib. Spec. 2007, 43, 140-151.

[8] M. S. Hamdy, O. Berg, J. C. Jansen, Th. Maschmeyer, J. A. Moulijn, G. Mul, Chem. Eur. J. 2006, 12, 620-628.

[9] Heikkila, T, Salonen, J., Tuura, J., Hamdy, M. S., Mul, G, Kumar, N., Salmi, T., Murzin, D. Y., Laitinen, L., Kaulonen, A. M., Hirvonen, J., and Lehto, V. P. Int. J. Pharmaceutics 2007, 331, 133-138.

[10] Hess, C.; Schlögl, R. Chem. Phys. Lett. 2006, 432, 139-145.

[11] Yeom, Y. H.; Frei, H. J. Phys. Chem. A 2002, 106, 3350-3355.

[12] Wasylenko, W.A.; Frei, H. Phys. Chem. Chem. Phys. 2007, 9, 5497-5502.

[13] Gisler, A.; Bürgi, T.; Baiker, A. Phys. Chem. Chem. Phys. 2003, 5, 3539-3548. 
[14] Landau, M.V.; Varkey, S.P.; Herskowitz, M.; Regev, O.; Pevzner, S.; Sen, T.; Luz, Z. Microporous Mesoporous Mater. 1999, 33, 149.

[15] Burgi, T.; Baiker, A. Adv.Catal. 2006, 50, 227.

[16] Socrates, G. Infrared and Raman Characteristic Group Frequencies, $3^{\text {rd }}$ Ed.; Wiley: New York, 2001.

[17] Magg, N.; Immaraporn, B.; Giorgi, J. B. ; Schroeder, T.; Bäumer, M.; Döbler, J.; Wu, Z.; Kondratenko, E.; Cherian, M.; Baerns, M.; Stair, P.C.; Sauer, J.; Freund, H.-J. J. Catal. 2004, 226, 88-100.

[18] Sheldon, R.A.; Kochi, J.K. Metal-Catalyzed Oxidations of Organic Compounds; Academic Press: New York, 1981.

[19] Busca, G.; Marchetti, L. J. Chem. Soc. Faraday Trans. 1985, 81, 1003-1014.

[20] Lempers, H., Delft University of Technology, 1998, PhD Thesis

[21] Lyons, J. E. Aspects of Homogenous Catalysis; Ugo, R., Ed.; Reidel: Dortrecht, 1977. p. 1-125.

[22] Keller, D.E,; de Groot, F.M.F.; Koningsberger, D.C.; Weckhuysen, B.M. J. Phys. Chem. B 2005, 109, 10223-10233.

[23] Wang, C.B.; Deo, G.; Wachs, I. E. J. Catal. 1998, 178, 640-648.

[24] Takenaka, S.; Tanaka, T.; Yamazaki, T.; Funabiki, T.; Yoshida, S. J. Phys. Chem. B 1997, 101, 9035-9040. 


\section{Figure Captions}

Figure 1: (a) ATR-FT-IR spectrum of V-TUD-1(2\%) before exposure to cyclohexene. Spectrum was referenced against neat diamond plate. (b) Difference spectrum recorded after evacuation of V-TUD-1 shown in trace (a). (c) Spectrum following exposure of V-TUD-1 to 50 Torr cyclohexene vapor, referenced against spectrum (b). The insert shows the UV-Vis spectrum of the hydrated catalyst, including an indication of the wavelength (458 nm) used for initiation of the reaction.

Figure 2: $\quad$ Steady state FT-IR spectroscopy of $458 \mathrm{~nm}$ induced cyclohexene $+\mathrm{O}_{2}$ photoreaction in V-TUD-1 silica sieve. Spectra were recorded after 30, 60, 90, and $120 \mathrm{~s}$ irradiation at $160 \mathrm{~mW}$ and are referenced against the spectrum before photolysis. The bottom trace shows an authentic spectrum of liquid cyclohexenone adsorbed on V-TUD-1 catalyst. A cyclohexene spectrum was subtracted to simulate the difference spectrum of cyclohexene to cyclohexenone oxidation.

Figure 3: $\quad$ Rapid scan spectra of $458 \mathrm{~nm}$ induced cyclohexene $+\mathrm{O}_{2}$ photoreaction in $\mathrm{V}$ TUD-1 silica sieve at RT. Times refer to the delay between the start of the photolysis pulse and the midpoint of the corresponding interferogram slice. The photolysis pulse duration was $480 \mathrm{~ms}$. Spectral resolution: $4 \mathrm{~cm}^{-1}$.

Figure 4: $\quad$ Rapid scan spectra of $458 \mathrm{~nm}$ induced cyclohexene $+\mathrm{O}_{2}$ photoreaction in $\mathrm{V}$ TUD-1 silica sieve, time period from $2 \mathrm{~s}$ to $10 \mathrm{~s}$ after the photolysis pulse. Times refer to delay between the start of the $480 \mathrm{~ms}$ photolysis pulse and the midpoint of the corresponding interferogram slice.

Figure 5: $\quad$ Proposed mechanism of cyclohexene $+\mathrm{O}_{2}$ photocatalysis over V-TUD-1 silica sieve. 